PREPARED FOR SUBMISSION TO JINST

$24^{\text {TH }}$ International Congress on X-ray Optics and Microanalysis

25-29 SEPTEMBER 2017

TRIESTE, ITALY

\title{
Beam tracking phase tomography with laboratory sources
}

\section{Fabio A. Vittoria, Marco Endrizzi, Gibril K. N. Kallon, Charlotte K. Hagen, Paul C. Diemoz, Anna Zamir, and Alessandro Olivo}

Department of Medical Physics and Biomedical Engineering, University College London, Malet Place, Gower Street, London WC1E 6BT, United Kingdom

E-mail: fabio.vittoria.12@ucl.ac.uk

\begin{abstract}
An x-ray phase-contrast laboratory system is presented, based on the beam-tracking method. Beam-tracking relies on creating micro-beamlets of radiation by placing a structured mask before the sample, and analysing them by using a detector with sufficient resolution. The system is used in tomographic configuration to measure the three dimensional distribution of the linear attenuation coefficient, difference from unity of the real part of the refractive index, and of the local scattering power of specimens. The complementarity of the three signals is investigated, together with their potential use for material discrimination.
\end{abstract}

Keywords: Computerized Tomography (CT) and Computed Radiography (CR), Multi-modality systems, X-ray radiography and digital radiography (DR), Inspection with X-rays 


\section{Contents}

1 Introduction 1

2 Beam tracking method 2

3 Experimental implementation $\quad 4$

4 Results and discussion $\quad 4$

5 Conclusions $\quad 6$

\section{Introduction}

X-rays are a form of electromagnetic radiation characterised by relatively high energy ( $\gtrsim 1 \mathrm{keV})$ and short wavelength $(\lesssim 1 \mathrm{~nm})$. They are routinely used for imaging in a variety of applications, from medical imaging to material science and security. X-rays are partially attenuated when travelling through matter, and the amount of attenuation depends on the sample's density, thickness and chemical composition. Due to their wave nature, $\mathrm{x}$-rays are not only attenuated in matter, but they also experience a phase shift. Since x-ray detectors are only sensitive to the intensity of the radiation impinging on them, standard x-ray imaging systems are insensitive to phase variations induced by a sample, and only rely on attenuation to generate contrast. Attenuation and phase effects can be described in terms of the complex refractive index of a material $n=1-\delta+i \beta$, where $\delta$ and $\beta$ are real, positive parameters, and $i$ is the imaginary unit. The parameter $\beta$ is responsible for attenuation, while $\delta$ describes phase effects. In the last decades, many efforts have been dedicated to building and developing $\mathrm{x}$-ray imaging methods that are not only sensitive to attenuation, but also to phase effects [1-13].

These x-ray phase-contrast imaging (XPCI) methods can be of great importance for all those applications in which attenuation contrast is low (i.e. when a particular sample provides low $\mathrm{x}$-ray attenuation, or when there is a small difference in attenuation between two different materials). Another advantage of XPCI is that attenuation and phase shift are two independent quantities, and the ability to measure both at the same time can be of particular importance for material discrimination. In this regard, a recent advance in XPCI is the possibility to measure a third signal, alongside attenuation and phase, called ultra-small-angle x-ray scattering (USAXS) [14]. X-ray scattering is generated by inhomogeneities in the spatial distribution of the electron density within a sample [15]. The angle at which $\mathrm{x}$-rays are scattered is related to the length scale at which the electron density distribution varies and to the wavelength of the radiation. The USAXS signal measured with XPCI methods is usually sensitive to inhomogeneities on length scales below the resolution of the imaging system, which typically means micrometric and sub-micrometric scale.

Although XPCI presents several advantages with respect to standard attenuation-based x-ray imaging, its implementation with laboratory sources usually requires the use of several optical 


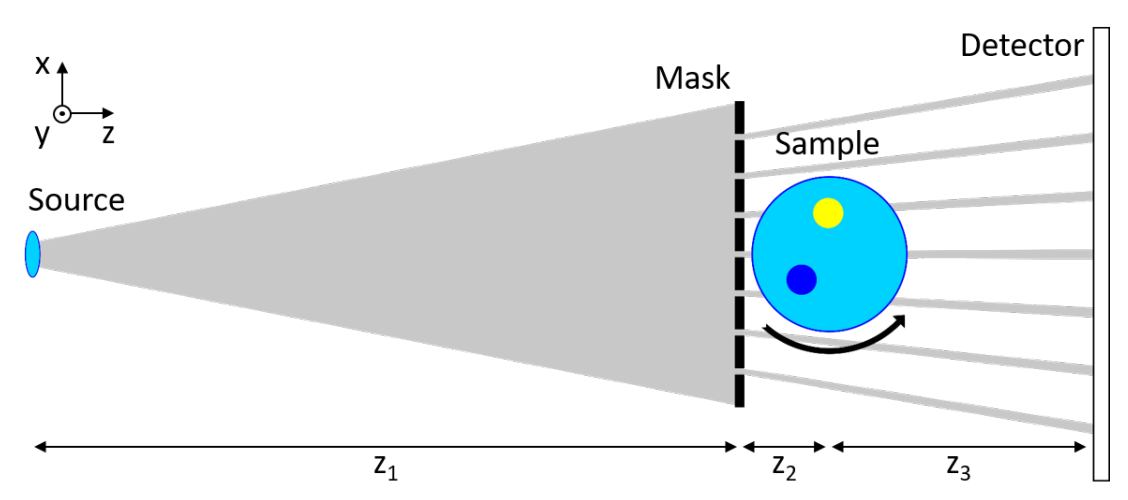

Figure 1. Schematic of the beam tracking setup.

elements, which increase the complexity of the experimental setup, introduce additional sources of error (e.g. relative alignment of the optical elements), and reduce the dose efficiency of the system $[16,17]$. The above problems are mostly solved by the beam tracking method [18-21]. In beam tracking an absorbing mask is used to create micrometric beamlets of radiation that, after propagating in free space, reach a detector where their spatial intensity distribution is measured. When a sample is introduced in the system as in Fig. 1, the intensity profile of each beamlet is modified. In particular, the total intensity of each beamlet decreases due to attenuation; the direction of propagation of each beamlet is modified by refraction, with the refraction angle being proportional to the spatial derivative of the phase shift induced by the sample; the divergence of each beamlet is increased due to USAXS. Beam tracking can be easily implemented with polychromatic laboratory sources, it is sensitive to sample attenuation, phase and USAXS, and requires only one optical element, placed before the sample. In this manuscript we show the results of a laboratory micro-tomography setup based on beam tracking, and how the three detected signals can be used for more effective material discrimination.

\section{Beam tracking method}

A schematic of the beam tracking setup is shown in Fig. 1. An absorbing mask is used to intercept the radiation emitted from an $\mathrm{x}$-ray source. The mask is characterised by a highly absorbing material featuring periodically repeated apertures though which $\mathrm{x}$-rays are transmitted. Each aperture extends along the $y$ direction, creating laminar beamlets that travel through the sample and, after propagation in free space, reach a detector.

Let $T_{M}(x)$ be the intensity transmission function of the absorbing mask, so that if $I_{0}(x, y)$ is the intensity distribution of the radiation impinging on the mask, $I_{1}(x, y)=T_{M}(x) I_{0}(x, y)$ is the intensity distribution just after the mask. Since the mask structures extend along the $y$ direction, $T_{M}$ depends only on the $x$ coordinate. Assuming that the effects of diffraction are negligible, so that the system can be described in terms of geometrical optics, and in the case of a point source, the intensity recorded at the detector, when no sample is present in the beam, is proportional to $I_{1}\left(x / M_{M}, y / M_{M}\right)$, where $M_{M}=\left(z_{1}+z_{2}+z_{3}\right) / z_{1}$ is the geometrical magnification between mask and detector plane, and $z_{1}, z_{2}$, and $z_{3}$ are the source to mask, mask to sample, and sample to 
detector distances, respectively. In the case of an extended source, described by a source intensity distribution $P(x, y)$, the intensity recorded at the detector, when no sample is present in the beam, is proportional to $I_{1}\left(x / M_{M}, y / M_{M}\right) * P\left(x /\left(M_{M}-1\right), y /\left(M_{M}-1\right)\right)$, where $*$ indicates the convolution operator. If the lateral extent of the projected source intensity distribution is larger than the distance between two adjacent beamlets (equal to the mask period multiplied by $M_{M}$ ), the beamlets overlap and the beam tracking method can no longer be applied. This is the first important condition for the implementation of beam tracking with extended sources. If this condition is met, each beamlet created by the mask is independent from the others. Beam tracking relies on the analysis of the variations induced by the sample to each beamlet. This can be achieved only if the detector has enough resolution to resolve the intensity profile of each beamlet. This is the second important condition for the implementation of beam tracking. If both the above conditions are satisfied, individual beamlets and their corresponding intensity profiles as recorded by the detector can be considered independently from one another.

Let us consider a single row of detector pixels for a certain position $y=y_{0}$. For simplicity we will omit the dependency on $y$ hereafter. Let $G(x)$ be the intensity profile of a single beamlet as recorded by the detector. $G(x)$ can usually be described by a Gaussian function [19]:

$$
G(x)=\frac{a}{\sqrt{2 \pi c^{2}}} \exp \left[-\frac{(x-b)^{2}}{2 c^{2}}\right]+d,
$$

where $a$ is the area of the Gaussian function, $b$ its central position, $c$ its standard deviation, and $d$ an additive constant. The parameter $d$ is used to describe partial transmission through the absorbing septa of the mask, the thickness of which can be insufficient to completely absorb the incoming radiation. When a sample is introduced in front of the aperture, the parameters $a, b, c$ and $d$ describing the beam intensity function $\mathrm{G}(\mathrm{x})$ are modified due to attenuation, phase shift and USAXS caused by the sample. In particular, attenuation decreases the total area $a$ of the Gaussian function, refraction (which is proportional to the first spatial derivative of the phase shift) varies its central position $b$, and USAXS increases its standard deviation $c$. From the variations of $a, b$ and $c$ it is possible to obtain the attenuation $(\mathrm{L})$, refraction $(\mathrm{R})$ and USAXS (S) signals:

$$
\begin{aligned}
& L=-\ln \left[a_{o} / a_{i}\right], \\
& R=\left(b_{o}-b_{i}\right) / z_{3}, \\
& S=\left(c_{o}^{2}-c_{i}^{2}\right) / z_{3}^{2},
\end{aligned}
$$

where the subscripts $o$ and $i$ refer to the parameters of $G(x)$ measured with and without the sample, respectively. It is important to note that the precision with which the refraction and USAXS signals are measured closely depend on the dimension of the intensity profiles of the beamlets in air $\left(c_{i}\right)$. Decreasing $c_{i}$ would, in general, increase the precision of $R$ and $S . c_{i}$ depends on source size, mask aperture size, and detector line spread function. The precision of the retrieval procedure, however, also depends on the total photon statistic measured by the detector. Reducing source size and/or mask aperture size would result in lower x-ray flux at the detector, while an increase in detector resolution usually results in a decrease in detector efficiency; all the above cases would therefore result, for a given exposure time, in lower photon statistic being detected. 
The signals $L, R$ and $S$ can all be expressed as line integrals of fundamental properties of the sample [22-25], and be used in tomographic reconstructions to obtain three-dimensional maps of the sample linear attenuation coefficient $\mu$, difference from unity of the real part of the refractive index $\delta$, and variance of the local USAXS distribution $\sigma_{\phi}^{2}$, respectively.

\section{Experimental implementation}

The experimental setup features a Rigaku MM009 source, with a rotating tungsten anode, operated at $46 \mathrm{kVp}$ and $26 \mathrm{~mA}$ and with focal spot of about $70 \mu \mathrm{m}$ full width at half maximum. The mask has period and aperture size equal to $93 \mu \mathrm{m}$ and $9 \mu \mathrm{m}$, respectively. It is made of a $225 \mu \mathrm{m}$ gold layer on a $525 \mu \mathrm{m}$ silicon substrate, and it was manufactured by Microworks GmbH (Karlsruhe, Germany). The detector is a Photonic Science (Mountfield, UK) CCD camera with pixel size of $4.54 \mu \mathrm{m}, 1: 1$ optically bounded to a fibre-optic, on the front of which has been deposited a gadolinium oxysulphide $\mathrm{x}$-ray scintillator. The effective resolution of the detector is $\approx 9 \mu \mathrm{m}$. The distances between the different elements of the setup are: source to mask $z_{1}=168 \mathrm{~cm}$, mask to sample $z_{2}=4 \mathrm{~cm}$, and sample to detector $z_{3}=30 \mathrm{~cm}$.

We imaged a custom made sample consisting of a plastic monofilament and a pencil lead enclosed in rubber. This sample was chosen to prove the complementarity of the three signals measured with beam tracking, and their potential use for material discrimination.

A tomographic dataset is acquired by rotating the sample in the angular range $\left[0^{\circ}, 180^{\circ}\right]$, with angular step $0.25^{\circ}$, for a total of 721 projections. For each rotation angle, the signals reconstructed from a single acquired image are sampled in the $x$ direction at a step equal to the mask period magnified at the sample plane, in this case $95 \mu \mathrm{m}$. In the $y$ direction, the sampling step is determined by the detector demagnified pixel size, equal to $3.87 \mu \mathrm{m}$. To increase the sampling rate in the $x$ direction, a 10-step scan of the sample is performed at each rotation angle, with a step size equal to $9.5 \mu \mathrm{m}$. The final images are obtained by recombining all the 10 steps of the scan, leading to sampling steps equal to 9.5 and $3.87 \mu \mathrm{m}$ in the $x$ and $y$ direction, respectively. The exposure time for each scan step was $5 \mathrm{~s}$, resulting in a $50 \mathrm{~s}$ exposure time for each rotation angle for the images acquired with the sample. A series of 10 images, each with $5 \mathrm{~s}$ exposure time, without the sample in the beam are acquired after every 20 angular projection, averaged, and used as reference. The detector dark current is measured by acquiring and averaging 20 frames with the source turned off, each with $5 \mathrm{~s}$ exposure time, 10 before and 10 after the tomographic scan. The detector dark current is subtracted from all the measurements acquired with and without the sample.

The parameters $a, b, c$ and $d$ in Eq. 2.1 are found through a non-linear least square minimization between the measured intensity profiles and the model function in Eq. 2.1. Tomographic reconstructions are performed with a standard filtered back projection algorithm; in the case of the attenuation and USAXS signals, the Ram-Lak filter used in the filtered back projection is multiplied by a Hamming window to reduce high frequency noise.

\section{Results and discussion}

Figure 2 shows the three reconstructed signals for a slice of the sample in the $x-z$ plane. These slices are obtained from the average of 27 detector rows in the $y$ direction, resulting in a voxel size 


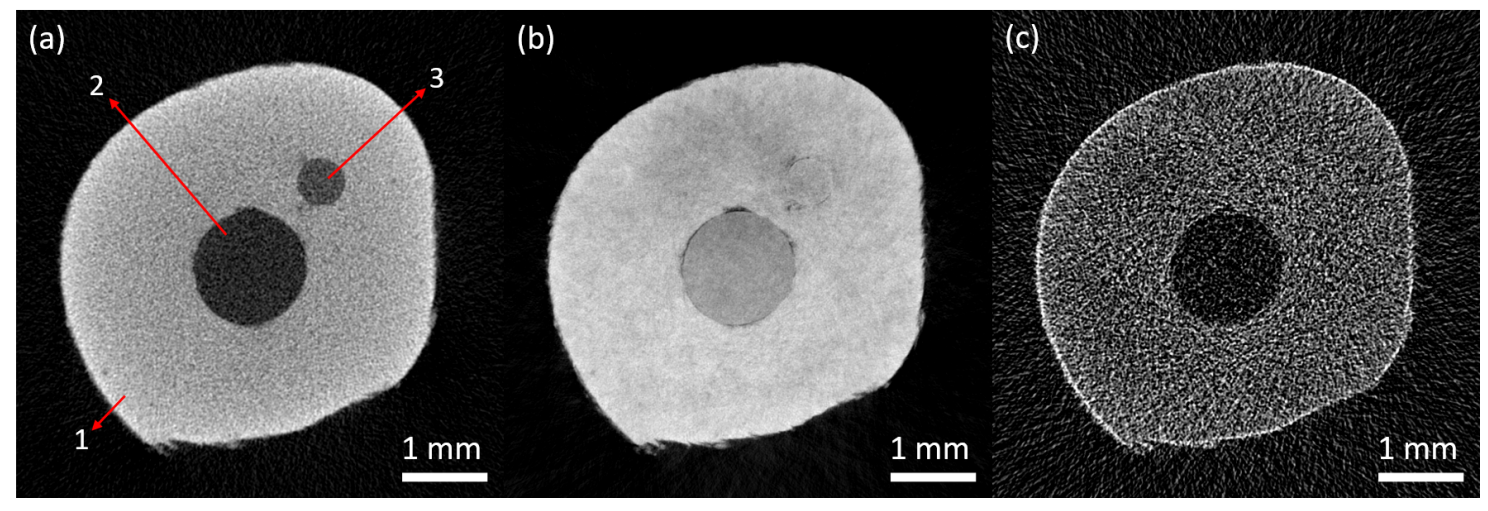

Figure 2. Reconstructed slices from the test sample made of rubber (1), plastic (2) and pencil lead (3). (a) Linear attenuation coefficient $\mu$; the displayed dynamic range is $[0,6] \mathrm{cm}^{-1}$. (b) Difference from unity of the real part of the index of refraction $\delta$; the displayed dynamic range is $\left[0,8 \times 10^{-7}\right]$. (c) Variance of the local USAXS distribution $\sigma_{\phi}^{2}$; the displayed dynamic range is $\left[0,4 \times 10^{3}\right] \mu \mathrm{rad}^{2} \mathrm{~cm}^{-1}$.

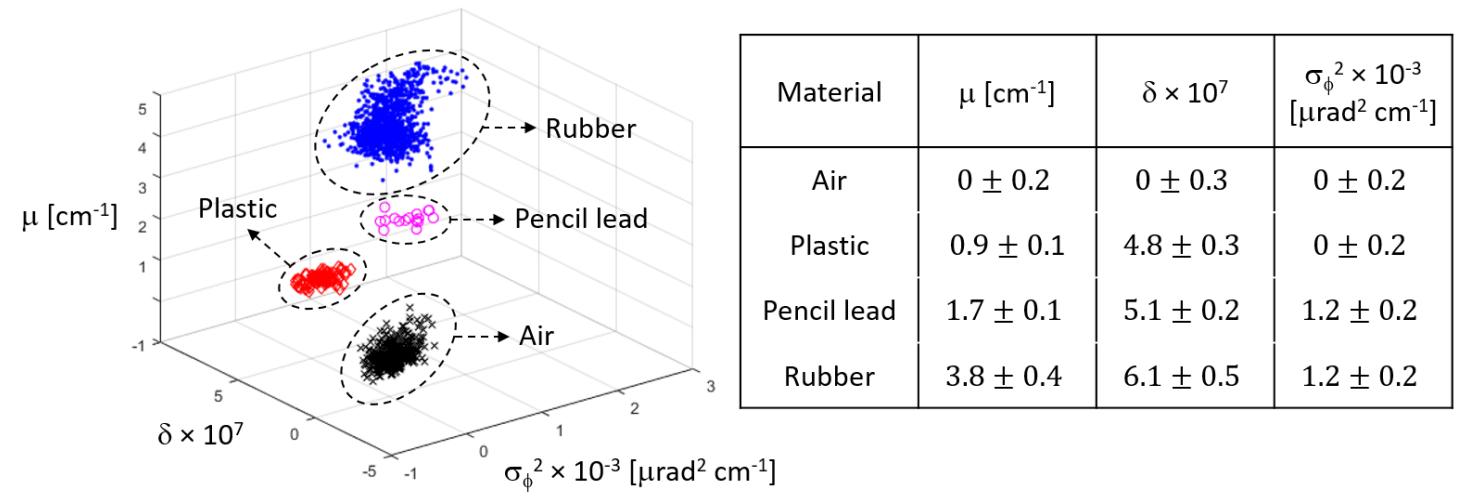

Figure 3. Scatter plot of $\mu, \delta$, and $\sigma_{\phi}^{2}$ for the materials in Fig. 2. The table on the right shows the mean values and standard deviations of the data in the scatter plot.

of $9.5 \times 105 \times 9.5 \mu \mathrm{m}^{3}$ in the $x, y$, and $z$ directions, respectively. In the attenuation image (Fig. 2 (a)) rubber can easily be distinguished from the other two materials, due to its higher attenuation coefficient, but the plastic and pencil lead samples provide similar contrast. In the phase image (Fig. 2 (b)), the signal to noise ratio for all materials is substantially higher than in the attenuation and USAXS images. However, while the plastic monofilament is clearly visible, rubber and pencil lead show a very similar signal, making it difficult to distinguish the two materials. The USAXS image (Fig. 2 (c)) shows a strong signal generated by rubber and pencil lead, due to their porosity. The composition of those samples is not homogeneous at length scales comparable to the resolution of the system, and this inhomogeneity creates the USAXS signal. Rubber and pencil lead, however, provide an almost identical USAXS signal, and it is therefore not possible to distinguish the two materials from Fig. 2 (c) only.

To better appreciate the complementarity of the three images, a scatter plot of the reconstructed signals is shown in Fig. 3, together with a table showing the average values of the retrieved signals and their corresponding standard deviations. Data displayed in Fig. 3 are obtained from the slices 
in Fig. 2, binned to a voxel size of $105^{3} \mu \mathrm{m}^{3}$. The images are manually segmented to isolate the different materials, and voxels at the interface between different materials, where the signals mix due to limited resolution, are discarded. Each material results in a different cluster in the scatter plot, and all clusters are well separated. The ability to measure three signals simultaneously can be of primary importance for material discrimination. Plastic and air, for example show negligible USAXS signal, but can be easily separated from the phase signal. Rubber and pencil lead, instead, present similar phase and USAXS signals, but different attenuation. Similar considerations apply for other combinations of materials and signals.

The current implementation of the method presents, however, some limitations. Noise in the retrieved USAXS signal is relatively high. If, for example, we consider the values retrieved for the pencil lead in the table in Fig. 3, the signal to noise ratio for the USAXS signal is 6, compared to 17 and 26 for the attenuation and phase signal, respectively. It is important to note that those values are referred to the signals binned to a voxel size of $105^{3} \mu \mathrm{m}^{3}$, and that high frequency noise is reduced for the attenuation and USAXS signal by the Hamming window used in the tomographic reconstruction. Another limitation is the total exposure time, and this is mainly limited by the 10-step scan which is performed at each projection to fully exploit the intrinsic resolution of the system.

\section{Conclusions}

We showed how the beam-tracking approach can be used to build a multi-modal laboratory micro-CT system, sensitive to attenuation, phase and USAXS. The possibility to simultaneously measure three different and independent physical properties can substantially facilitate material discrimination in a given sample. In this work we show how signals from different materials are well separated in the three-dimensional space $\left(\mu, \delta, \sigma_{\phi}^{2}\right)$, suggesting the possibility to use a simple segmentation method based on thresholds in this three-dimensional space. Ultimately, the ability to discriminate two different materials depends on the noise level in the reconstructed images, but it is important to note that, for a given level of noise in the attenuation signal, the additional information obtained from the phase and USAXS signal are always beneficial compared to a standard attenuation based $\mathrm{x}$-ray system. Future developments might include the use of advanced image fusion methods, in which the three signals are combined together in a single image with higher information content. Finally, alternative acquisition schemes and data analysis procedures will be studied to mitigate the problems related to the high noise in the retrieved USAXS signal and to reduce the total scan time.

\section{Acknowledgments}

This work is funded by the EPSRC (Grants EP/M507970/1 and EP/I021884/1). ME was supported by the Royal Academy of Engineering under the RAEng Research Fellowships scheme.

\section{References}

[1] S. W. Wilkins, Ya. I. Nesterets, T. E. Gureyev, Mayo S. C., A. Pogany, and Stevenson A. W. On the evolution and relative merits of hard x-ray phase-contrast imaging methods. Phil. Trans. R. Soc. A, 372:20130021, 2014. 
[2] A. Olivo and E. Castelli. X-ray phase contrast imaging: From synchrotrons to conventional sources. Riv. Nuovo Cimento, 37:467-508, 2014.

[3] U. Bonse and M. Hart. An x-ray interferometer. Appl. Phys. Lett., 6:155-156, 1965.

[4] A. Momose, T. Takeda, Y. Itai, and K. Hirano. Phase-contrast X-ray computed tomography for observing biological soft tissues. Nat. Med., 2:473-475, 1996.

[5] A. Snigirev, I. Snigireva, V. Kohn, S. Kuznetsov, and I. Schelokov. On the possibilities of x-ray phase contrast microimaging by coherent high-energy synchrotron radiation. Rev. Sci. Instrum., 66:5486-5492, 1995.

[6] S. W. Wilkins, T. E. Gureyev, D. Gao, A. Pogany, and A. W. Stevenson. Phase-contrast imaging using polychromatic hard x-rays. Nature, 384:335-338, 1996.

[7] V. N. Ingal and E. A. Beliaevskaya. X-ray plane-wave topography observation of the phase contrast from a non-crystalline object. J. Phys. D, 28:2314-2317, 1995.

[8] D. Chapman, W. Thomlinson, R. E. Johnston, E. Washburn, D. ans Pisano, N. Gmür, Z. Zhong, R. Menk, F. Arfelli, and D. Sayers. Diffraction enhanced x-ray imaging. Phys. Med. Biol., 42:2015-2025, 1997.

[9] C. David, B. Nohammer, H. H. Solak, and E. Ziegler. Differential x-ray phase contrast imaging using a shearing interferometer. Appl. Phys. Lett., 81:3287-3289, 2002.

[10] A. Momose, S. Kawamoto, I. Koyama, Y. Hamaishi, K. Takai, and Y. Suzuki. Demonstration of x-ray talbot interferometry. Jpn. J. Appl. Phys., 42:L866-L868, 2003.

[11] F. Pfeiffer, T. Weitkamp, O. Bunk, and C. David. Phase retrieval and differential phase-contrast imaging with low-brilliance X-ray sources. Nat. Phys., 2:258-261, 2006.

[12] A. Olivo, F. Arfelli, G. Cantatore, R. Longo, R. H. Menk, S. Pani, M. Prest, P. Poropat, L. Rigon, G. Tromba, E. Vallazza, and E. Castelli. An innovative digital imaging set-up allowing a low-dose approach to phase contrast applications in the medical field. Med. Phys., 28:1610-1619, 2001.

[13] A. Olivo and R. Speller. A coded-aperture approach allowing x-ray phase contrast imaging with conventional sources. Appl. Phys. Lett., 91:074106, 2007.

[14] E. Pagot, P. Cloetens, S. Fiedler, A. Bravin, P. Coan, J. Baruchel, J. Härtwig, and W. Thomlinson. A method to extract quantitative information in analyser-based x-ray phase contrast imaging. Appl. Phys. Lett., 82:3421, 2003.

[15] O. Glatter and O. Kratky. Small Angle X-ray Scattering. Academic Press, 1982.

[16] F. Pfeiffer, M. Bech, O. Bunk, P. Kraft, E. F. Eikenberry, C. Brönnimann, C. Grünzweig, and C. David. Hard-x-ray dark-field imaging using a grating interferometer. Nat. Mater., 7:134, 2008.

[17] M. Endrizzi, P. C. Diemoz, T. P. Millard, J. Louise Jones, R. D. Speller, I. K. Robinson, and A. Olivo. Hard X-ray dark-field imaging with incoherent sample illumination. Appl. Phys. Lett., 104:024106, 2014.

[18] F. A. Vittoria, M. Endrizzi, P. C. Diemoz, U. H. Wagner, C. Rau, I. K. Robinson, and A. Olivo. Virtual edge illumination and one dimensional beam tracking for absorption, refraction, and scattering retrieval. Appl. Phys. Lett., 104:134102, 2014.

[19] F. A. Vittoria, G. K. N. Kallon, D. Basta, P. C. Diemoz, I. K. Robinson, A. Olivo, and M. Endrizzi. Beam tracking approach for single-shot retrieval of absorption, refraction and dark-field signals with laboratory x-ray sources. App. Phys. Lett., 106:224102, 2015. 
[20] F. A. Vittoria, M. Endrizzi, P. C. Diemoz, A. Zamir, U. H. Wagner, C. Rau, I. K. Robinson, and A. Olivo. X-ray absorption, phase and dark-field tomography through a beam tracking approach. Sci. Rep., 5:16318, 2015.

[21] F. A. Vittoria, M. Endrizzi, G. K. Kallon, C. K. Hagen, F. Iacoviello, P. De Coppi, and A. Olivo. Multimodal phase-based x-ray microtomography with nonmicrofocal laboratory sources. Phys. Rev. Applied, 8:064009, 2017.

[22] M. N. Wernick, O. Wirjadi, D. Chapman, Z. Zhong, N. P. Galatsanos, Y. Yang, J. G. Brankov, O. Oltulu, M. A. Anastasio, and C. Muehleman. Multiple-image radiography. Phys. Med. Biol., 48:3875-3895, 2003.

[23] M. Bech, O. Bunk, T. Donath, R. Feidenhans'l, C. David, and F. Pfeiffer. Quantitative x-ray dark-field computed tomography. Phys. Med. Biol., 55:5529-5539, 2010.

[24] L. Rigon, A. Astolfo, F. Arfelli, and R. Menk. Generalized diffraction enhanced imaging: Application to tomography. Eur. J. Radiol., 68S:S3-S7, 2008.

[25] F. A. Vittoria, M. Endrizzi, and A. Olivo. Retrieving the ultrasmall-angle x-ray scattering signal with polychromatic radiation in speckle-tracking and beam-tracking phase-contrast imaging. Phys. Rev. Applied, 7:034024, 2017. 